\title{
Comparative evaluation of unfixed and fixed human neutrophils for determination of antineutrophil cytoplasmic antibodies by indirect immunofluorescence
}

Medicine, Division of Gastroenterology, Örebro Medical Centre Hospital, S-701 85, Örebro, Sweden.

Accepted for publication 13 May 1997

\begin{abstract}
Background-Antineutrophil cytoplasmic antibodies (ANCAs) are found in the sera of patients with vasculitides and ulcerative colitis. Using indirect immunofluorescence on ethanol fixed neutrophils, ANCAs can be divided into two types: those that give a cytoplasmic staining pattern (C-ANCA) and those that give a perinuclear staining pattern (P-ANCA). Some studies have indicated that the perinuclear staining pattern might be an artefact of alcohol fixation.
\end{abstract}

Aims-To observe any changes seen in the ANCA staining pattern using indirect immunofluorescence on unfixed neutrophils or neutrophils that had been fixed by ethanol, acetone, or paraformaldehyde. In addition, the effects of the different fixation methods on the sensitivity of the indirect immunofluorescence test were evaluated.

Methods-Twenty one sera from patients with ulcerative colitis and 19 from healthy controls were studied. In addition, 17 sera from patients with vasculitides, including eight with proteinase 3 (PR 3) positive C-ANCA and nine with myeloperoxidase (MPO) positive P-ANCA were included in the study. Antineutrophil cytoplasmic antibodies were analysed by indirect immunofluorescence on unfixed neutrophils or cells fixed by ethanol, acetone, or paraformaldehyde.

Results-All the ulcerative colitis associated ANCA positive sera presented a perinuclear staining pattern on both unfixed and fixed cells. The acetone and paraformaldehyde fixations decreased the ulcerative colitis P-ANCA titres. Paraformaldehyde fixation also decreased the MPO positive P-ANCA titres. In indirect immunofluorescence, the staining patterns of all the eight PR 3 positive C-ANCA sera and eight of the nine MPO positive P-ANCA sera did not change, even if unfixed or fixed cells were used.

Conclusions-The ANCA staining patterns are not affected by the fixation method used, and are the same as when unfixed neutrophils are used; this suggests that the P-ANCA pattern is not an artefact of alcohol fixation. Furthermore, this study confirms that on ethanol fixed neutrophils, the antigen of ulcerative coli- tis associated P-ANCA is better exposed than on acetone or paraformaldehyde fixed cells.

(F Clin Pathol 1997;50:677-680)

Keywords: antineutrophil cytoplasmic antibodies; fixation methods; indirect immunofluorescence

Antineutrophil cytoplasmic antibodies (ANCAs) were described originally in sera from patients with Wegener's granulomatosis and other forms of vasculitides. ${ }^{1-3}$ Later, they were found also in patients with inflammatory bowel diseases. ${ }^{4}$ The standard method for detection of ANCAs is indirect immunofluorescence on ethanol fixed neutrophils, as defined by two international workshops. ${ }^{56}$ Using this technique, ANCAs give two distinct patterns - a cytoplasmic staining pattern (CANCA) or a perinuclear staining pattern (P-ANCA). ${ }^{7}$ The cytoplasmic staining pattern was found to be strongly associated with Wegener's granulomatosis, ${ }^{2}$ whereas the perinuclear staining pattern was connected with systemic vasculitis and inflammatory bowel disease. ${ }^{34}$ The antigen of the cytoplasmic staining pattern associated with Wegener's granulomatosis has been identified as proteinase 3 (PR 3). ${ }^{89}$ The antigen of $\mathrm{P}$-ANCA in most patients with other vasculitides has been shown to be myeloperoxidase (MPO), ${ }^{310}$ whereas the antigen of P-ANCA in patients with ulcerative colitis has not been defined. ${ }^{112}$

It has been reported that the anti-MPO associated P-ANCA reaction in vasculitides is an artefact caused by alcohol fixation of the neutrophils, which causes cytoplasmic antigens to redistribute around the nucleus. ${ }^{3}$ In ulcerative colitis, some studies ${ }^{13-15}$ have shown that the P-ANCA reaction also might be an artefact of alcohol fixation, but others studies have not supported this hypothesis. ${ }^{416}$

Regarding the sensitivity of the test, Falk and Jennette ${ }^{3}$ reported that, in patients with vasculitis, formalin fixation did not alter the binding of ANCAs to neutrophils when compared to ethanol fixation. In ulcerative colitis, Cambridge $e t ~ a l^{17}$ found that the paraformaldehyde and acetone fixations significantly reduced the reactivity of ANCAs with neutrophils.

The aim of the present study was to evaluate how various neutrophil fixation methods influenced the indirect immunofluorescence ANCA staining pattern in patients with a positive P-ANCA or C-ANCA in comparison to stand- 
ard methodology (ethanol fixed neutrophils). In addition, the effect of fixation methods on the sensitivity of the test was also investigated.

\section{Methods}

SERUM SPECIMENS AND STUDY SUBJECTS

Twenty one serum samples from 21 patients with ulcerative colitis (nine women, 12 men; median age 45.5 years, range 22-74 years) were included in the study. The diagnostic criteria were consistent with those in a previous study. ${ }^{18}$

In addition, 17 ANCA positive sera from patients with small vessel vasculitides, nine with anti-MPO positive P-ANCA, and eight with anti-PR 3 positive C-ANCA were used to test the influence of different fixation methods. The anti-MPO and anti-PR 3 activities of the sera were determined by enzyme immunoassay kits for quantitative determination of antibodies to MPO and PR 3 (Ferring Diagnostica AB, Malmö, Sweden). The P-ANCA and C-ANCA staining patterns were screened by indirect immunofluorescence with ethanol fixed neutrophils.

Nineteen serum samples from blood donors (seven women, 12 men; median age 46.0 years, range $22-63$ years) were used as healthy controls.

\section{PREPARATION OF HUMAN NEUTROPHILS}

Heparinised venous blood was drawn from healthy blood donors and staff. Neutrophils were separated by Ficoll-Paque (Pharmacia Biotech AB, Uppsala, Sweden) density centrifugation according to the method of Bøyum. ${ }^{19}$ Glass slides displaying neutrophils on a well defined area were prepared by means of cytocentrifugation (Shandon Cytospin, Cheshire, UK).

\section{DIFFERENT FIXATION METHODS}

Unfixed neutrophils were stored at $4^{\circ} \mathrm{C}$ and tested within one week of preparation. Ethanol fixed neutrophils were fixed in $99 \%$ ethanol for 5-10 minutes at $4^{\circ} \mathrm{C}$, air dried, and stored at $4^{\circ} \mathrm{C}$ until use. ${ }^{35}$ Acetone fixed neutrophils were fixed in absolute acetone for 30-60 seconds at room temperature, air dried, and stored at $4^{\circ} \mathrm{C}$ until use.${ }^{17}$ Paraformaldehyde fixed neutrophils were fixed in $0.5 \%$ paraformaldehyde for 10 minutes at room temperature followed by absolute acetone at $-20^{\circ} \mathrm{C}$ for one minute, air dried, and stored at $-20^{\circ} \mathrm{C}$ until use. ${ }^{16}$

\section{DEMONSTRATION OF ANCAS BY INDIRECT} IMMUNOFLUORESCENCE

Sera from patients were diluted $1 / 10$ in

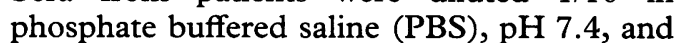
then tested for the presence of ANCAs. Approximately $33 \mu$ lof the diluted serum was applied on to an area of a glass slide displaying a well defined layer of neutrophils and incubated in a humid chamber for 30 minutes at room temperature. After washing with PBS for 10 minutes and incubation for another 30 minutes with a $1 / 10$ dilution of fluorescein isothiocyanate (FITC) labelled $\mathrm{F}(\mathrm{ab}) 2$ rabbit antihuman IgG antibodies (Dakopatts $\mathrm{A} / \mathrm{S}$, Copenhagen, Denmark), the slides were washed again in PBS for 10 minutes, and finally mounted under a coverslip with PBSglycerine. The slides were scrutinised at a magnification of $\times 250$ under a Zeiss epifluorescence microscope equipped with a $50 \mathrm{~W}$ tungsten lamp, BD $450-490$ as the primary filter and LP 520 as the barrier filter. Sera giving a typical perinuclear or cytoplasmic staining reaction with the neutrophils were regarded as positive. These sera were re-tested at the initial dilution of $1 / 10$ and further twofold dilutions of between $1 / 20$ and $1 / 640$.

DEMONSTRATION OF ANTINUCLEAR ANTIBODIES BY INDIRECT IMMUNOFLUORESCENCE

Standard techniques were used to demonstrate antinuclear antibodies (ANAs) with indirect immunofluorescence on slides with cryostat sections of rat liver tissue and HEp-2 cells.

\section{ANA TESTS ON THE CRYOSTAT SECTIONS OF RAT} LIVER TISSUES

Cryostat sections, $5 \mu \mathrm{m}$ thick, of rat liver tissue were placed on slides and fixed for 10 minutes at room temperature in absolute acetone, air dried, stored at $4^{\circ} \mathrm{C}$, and used within one week of preparation. Fluorescein isothiocyanate labelled $\mathrm{F}(\mathrm{ab}) 2$ rabbit antihuman IgG antibodies (see above) were used as the secondary antibody. Sera in the indirect immunofluorescence tests giving a typical homogeneous or speckled staining reaction with the cell nucleus of the rat liver tissues were regarded as positive. The serum samples were tested at an initial dilution of $1 / 10$ and further at twofold dilutions of between $1 / 20$ and $1 / 1280$.

ANA TESTS ON HEP-2 CELLS

The ANA kit with mitotic human epithelioid cells (HEp-2) for semiquantitative detection of ANAs in human serum by indirect immunofluorescence was purchased from Immuno Concepts (Sacramento, California, USA). The tests were performed according to the manufacturer's instructions.

To eliminate the influence of ANAs on ANCA analysis, only ANA negative sera were included in the present study.

\section{STATISTICAL ANALYSIS}

Cochran Q test, Friedman two-way analysis of variance by ranks, Wilcoxon signed ranks test and Spearman's rank correlation test were used for statistical analysis. A p value $<0.05$ was considered to be statistically significant.

\section{Results}

Using indirect immunofluorescence on ethanol fixed neutrophils, 13 of 21 (61.9\%) sera from patients with ulcerative colitis were found to be ANCA positive and all the 13 positive sera gave a perinuclear staining pattern. No cytoplasmic staining pattern was found in any of the ulcerative colitis patients.

When the test was performed on unfixed neutrophils or cells fixed in paraformaldehyde or acetone, the ulcerative colitis P-ANCA pattern did not change. However, one serum sample which was positive with a low titre of $1: 10$ when ethanol fixed neutrophils were used was 
Table 1 Summary of the titres of $P-A N C A$ in sera from patients with ulcerative colitis

Number of sera and distribution of P-ANCA titres with neutrophils fixed by

\begin{tabular}{|c|c|c|c|c|}
\hline \multirow[b]{2}{*}{$P-A N C A$ titres } & \\
\hline & Ethanol & Acetone & Paraformaldehyde & Unfixed \\
\hline $1: 320-1: 640$ & 3 & 1 & 0 & 3 \\
\hline $1: 160$ & 5 & 5 & 1 & 5 \\
\hline $1: 40-1: 80$ & 3 & 4 & 5 & 3 \\
\hline $1: 10-1: 20$ & 2 & 2 & 5 & 1 \\
\hline$<1: 10$ & 8 & 9 & 10 & 9 \\
\hline Median titre & $1: 40$ & $1: 20$ & $1: 10$ & $1: 40$ \\
\hline Range & $<1: 10-1: 640$ & $<1: 10-1: 320$ & $<1: 10-160$ & $<1: 10-1: 640$ \\
\hline No of positive patients (\%) & $13 / 21(61.9)$ & $12 / 21(57.1)$ & $11 / 21(52.4)$ & $12 / 21(57.1)$ \\
\hline
\end{tabular}

The titre of P-ANCA $<1: 10$ is considered as negative.

Table 2 Summary of the titres of anti-MPO positive $P-A N C A$ sera $(n=9)$

Number of sera and distribution of P-ANCA titres with neutrophils fixed by

\begin{tabular}{lllll}
\cline { 2 - 5 }$P$-ANCA titres & Ethanol & Acetone & Paraformaldehyde & Unfixed \\
\hline $1: 320-1: 640$ & 3 & 3 & 1 & 3 \\
$1: 160$ & 2 & 2 & 1 & 2 \\
$1: 40-1: 80$ & 4 & 4 & $6^{\star}$ & 4 \\
$1: 10-1: 20$ & 0 & 0 & 1 & 0 \\
Titres (median) & $1: 160$ & $1: 160$ & $1: 80$ & $1: 160$ \\
Range & $1: 80-1: 640$ & $1: 80-1: 640$ & $1: 20-1: 320$ & $1: 80-1: 640$ \\
\hline
\end{tabular}

*One of them presented as C-ANCA (titre, 1:40).

Table 3 Summary of the titres of anti-proteinase 3 positive $C-A N C A$ sera $(n=8)$

Number of sera and distribution of $C-A N C A$ titres with neutrophils fixed by

\begin{tabular}{|c|c|c|c|c|}
\hline$C-A N C A$ titres & Ethanol & Acetone & Paraformaldehyde & Unfixed \\
\hline $1: 320-1: 640$ & 1 & 1 & 1 & 1 \\
\hline $1: 160$ & 3 & 1 & 1 & 3 \\
\hline $1: 80$ & 3 & 5 & 3 & 3 \\
\hline $1: 40$ & 1 & 1 & 3 & 1 \\
\hline Titres (median) & $1: 120$ & $1: 80$ & $1: 80$ & $1: 120$ \\
\hline Range & $1: 40-1: 320$ & $1: 40-1.320$ & $1: 40-1: 320$ & $1: 40-1: 320$ \\
\hline
\end{tabular}

negative when unfixed, acetone fixed, or paraformaldehyde fixed neutrophils were used. One further serum sample which was positive when ethanol fixed neutrophils were used (titre, 1:20) was negative when studied with paraformaldehyde fixed neutrophils. In spite of this, the results correlated well when alternative fixation methods were compared with ethanol: $r=0.842-0.999, \mathrm{p}<0.0001$ (table 1). However, when acetone or paraformaldehyde fixed neutrophils were used the ulcerative colitis P-ANCA titres decreased for some patients $(p=0.0029$; table 1$)$. Because most of the ulcerative colitis P-ANCA positive sera (10 of 13) had high titres $(\geqslant 1: 80)$ when tested on ethanol fixed neutrophils, the sensitivity of the test was not changed significantly by different fixation methods, as they were still positive, although with a lower titre (table 1).

All nine MPO positive P-ANCA sera still gave a perinuclear staining pattern when unfixed neutrophils or cells fixed by acetone were used. Eight of the nine MPO positive $P$-ANCA expressed a perinuclear staining pattern on paraformaldehyde fixation but one gave a cytoplasmic staining pattern. Compared with ethanol fixed cells, the use of unfixed or acetone fixed cells did not influence the titres of MPO positive P-ANCA, while the use of paraformaldehyde fixed cells decreased the P-ANCA titres $(p=0.0169)$ (table 2).

All eight $P R 3$ positive C-ANCA sera presented a C-ANCA indirect immunofluorescence pattern on unfixed neutrophils, cells fixed by acetone, or cells fixed by paraformaldehyde. The titres of the PR 3 positive C-ANCA were not altered significantly by the different fixation methods $(p=0.35)$. The median titres and ranges are shown in table 3.

Serum samples from 19 blood donors were all ANCA negative in the indirect immunofluorescence test when the different fixation methods were used.

\section{Discussion}

Generally, fixation of biological cells can be achieved with organic solvents or cross linking reagents. Organic solvents such as alcohols and acetone remove lipids and dehydrate the cells, precipitating the proteins on the cellular architecture. Cross linking reagents such as paraformaldehyde form intermolecular bridges, normally through free amino groups, thus creating a network of linked antigens. ${ }^{20}$

Although fixation is used to prevent the occurrence of various artefacts, it may itself cause changes within the cell so that it deviates significantly from the normal. ${ }^{21}$ Therefore, it has been suggested by immunopathologists that unfixed cell preparations should be used whenever possible, unless the antigen under investigation is known to be soluble. ${ }^{22}$

To date, we have not found any reports analysing ANCAs on unfixed human neutrophils in an indirect immunofluorescence test. In the present study, we used not only ethanol, acetone, and paraformaldehyde fixed neutrophils, but also unfixed ones, to observe possible changes in the ANCA staining pattern. We found that all the $13 \mathrm{P}$-ANCA positive sera in patients with ulcerative colitis and eight out of the nine MPO positive P-ANCA sera gave a perinuclear pattern on both unfixed and fixed cells, while all the eight PR 3 positive C-ANCA sera expressed a cytoplasmic staining pattern on both unfixed and fixed cells, independent of the fixation method used. These findings suggest that the ANCA pattern is very stable and the same when both unfixed or fixed neutrophils are used. Thus, the ANCA pattern does not appear to be a result of an artefact of ethanol fixation, as has been suggested. This does not contradict the fact that various neutrophil fixation methods may influence the ANCA staining pattern. Falk and Jennette ${ }^{3}$ first reported that the P-ANCA expressed in MPO positive sera from patients with vasculitides, and demonstrated on ethanol fixed neutrophils, could be changed to a cytoplasmic staining pattern in formalin fixed neutrophils. This was thought to be owing to a redistribution of MPO from the azurophilic or primary granules in the cytosol to perinuclear sites as a result of ethanol fixation (electro-ionic charges), an effect that could be prevented by formalin fixation. However, Spickett and Broomhead $^{23}$ showed that formalin fixation gave inconsistent results.

Studies on antigen specificities of P-ANCA in ulcerative colitis have been inconsistent. Some investigators reported a correlation with autoantibodies against neutrophil cytoplasmic proteins, such as lactoferrin and $\beta$-glucuronidase, ${ }^{24} 25$ findings that have not 
been confirmed by others. ${ }^{11}{ }^{12} 26-29$ In addition, some studies indicated that the P-ANCA pattern in patients with ulcerative colitis was an artefact of alcohol fixation and that it could be converted to a cytoplasmatic pattern by non-alcohol fixation methods. ${ }^{13-15}$ However, other studies did not support these results, as they found that the perinuclear staining pattern in ulcerative colitis was maintained with various fixatives including paraformaldehyde, glutaraldehyde, and ethanol. ${ }^{416}$ Furthermore, our studies on unfixed neutrophils also showed a perinuclear staining pattern.

The disparity between previous studies could be due to methodological differences. Initially, we used neutrophils fixed in buffered formalin acetone solution to observe the influence of this method on the ANCA staining patterns. We found that some sera that were P-ANCA positive on ethanol fixed neutrophils gave a granular cytoplasmic staining pattern (C-ANCA) on neutrophils fixed in buffered formalin acetone, and others became negative, but this was not reproducible from batch to batch (results not shown). This is in agreement with the findings of Spickett and Broomhead. ${ }^{23}$ Furthermore, we found that formalin fixed neutrophils occasionally gave positive C-ANCA reactions with sera from healthy blood donors that could not be reproduced with unfixed or ethanol fixed neutrophils (results not shown). Using paraformaldehyde fixation, the P-ANCA pattern of sera from ulcerative colitis patients was not changed, which is in agreement with the results by Billing et al. ${ }^{16}$

We found that the acetone and paraformaldehyde fixations decreased the ulcerative colitis P-ANCA titres, but the sensitivity of the test remained mainly unchanged. Cambridge $e t ~ a l^{17}$ found that when ethanol fixed cells were used, only sera with a high titre $(>1: 50)$ remained positive on acetone fixed neutrophils and $60 \%$ of these sera lost their ANCA positivity when tested on paraformaldehyde fixed cells. This is consistent with our findings suggesting that the antigen(s) of ulcerative colitis P-ANCA is better exposed on ethanol fixed cells.

In conclusion, our results show that both the ulcerative colitis associated and anti-MPO associated P-ANCA patterns are expressed equally well on unfixed or ethanol fixed neutrophils; the same applies to C-ANCA. Thus, the P-ANCA pattern does not appear to be the result of an artefact caused by the fixation method. Furthermore, we confirm the results of the international workshops, ${ }^{56}$ showing that when the indirect immunofluorescence method is used to detect ANCAs, the best results are obtained with ethanol fixed human neutrophils.

Professors Dan Danielsson and Gunnar Järnerot kindly gave assistance, advice, and criticism during the performance of this study. Statistical advice was given by Associate Professor Lennart Brodin. Ping Yang was financially supported by Örebro Medical Centre Research Foundation.

1 Davies DJ, Moran JE, Niall JF, Ryan GB. Segmental necrotising glomerulonephritis with anti-neutrophil antibody: possible arbovirus aetiology? BMF 1982;285:606

2 Van der Woude FJ, Rasmussen N, Lobatto S, Wiik A, Permin H, Van Es LA, et al. Autoantibodies against neutrophils and monocytes: tool for diagnosis and marker of disease activity in Wegener's granulomatosis. Lancet 1985;1:425-9

3 Falk RJ, Jennette JC. Anti-neutrophil cytoplasmic autoantibodies with specificity for myeloperoxidase in patients with systemic vasculitis and idiopathic necrotizing and crescensystemic vasculitis and idiopathic necrotizing and crescen

4 Saxon A, Shanahan F, Landers C, Ganz T, Targan S. A distinct subset of antineutrophil cytoplasmic antibodies is tinct subset of antineutrophil cytoplasmic antibodies is
associated with inflammatory bowel disease. $\mathcal{f}$ Allergy Clin Immunol 1990;86:202-10.

5 Wiik A. Delineation of a standard procedure for indirect immunofluorescence detection of ANCA. APMIS 1989; 97(Suppl 6): 12-13

6 Hagen EC, Andrassy K, Chernok E, Daha MR, Gsakin G, Gross W, et al. The value of indirect immunofluorescence and solid phase techniques for ANCA detection. A report on the first phase of an international co-operative study on the standardisation of ANCA assays. F Immunol Methods 1993;159:1-16.

7 Wiik A, Van der Woude FJ. The new ACPA/ANCA nomenclature. Neth $\mathcal{F}$ Med 1990;36:107-8.

8 Lüdemann J, Utecht B, Gross WL. Anti-neutrophil cytoplasm antibodies in Wegener's granulomatosis recognize an elastinolytic enzyme. $\mathcal{F}$ Exp Med 1990;171:357-62.

9 Goldschmeding R, van der Schoot CE, ten Bokkel Huinink D, Hack CE, van den Ende ME, Kallenberg CGM, et al. Wegener's granulomatosis autoantibodies identify a novel diisopropylfluorophosphate-binding protein in the lysosomes of normal human neutrophils. $\mathcal{F}$ Clin Invest $1989 ; 84$ : 1577-87.

10 Jennette JC, Wilkman AS, Falk RJ. Anti-neutrophil cytoplasmic autoantibody-associated glomerulonephritis and vasculitis. Am $\mathcal{F}$ Pathol 1989;135:921-30.

11 Hertervig E, Wieslander J, Johansson C, Wiik A, Nilsson $\AA$. Anti-neutrophil cytoplasmic antibodies in chronic inflammatory bowel disease. Prevalence and diagnostic role. Scand F Gastroenterol 1995;30:693-8.

12 Yang P, Bohr J, Tysk C, Danielsson D, Järnerot G. Antineutrophil cytoplasmic antibodies in inflammatory bowel disease and collagenous colitis: no association with lactoferrin, $\beta$-glucuronidase, myeloperoxidase, or proteinase 3. Inflamm Bowel Dis 1996;2:173-7.

13 Hardarson S, LaBrecque DR, Mitros FA, Neil GA, Goeken JA. Antineutrophil cytoplasmic antibody in inflammatory bowel disease and hepatobiliary disease. Am $\mathcal{f}$ Clin Pathol 1993;99:277-81.

14 Mulder AHL, Broekroelofs J, Horst G, Limburg PC, Nelis GF, Kallenberg CGM. Anti-neutrophil cytoplasmic antibodies (ANCA) in inflammatory bowel disease: characterization and clinical correlates. Clin Exp Immunol 1994;95: 490-7.

15 Ellerbroek PM, Oudkerk Pool M, Ridwan BU, Dolman $\mathrm{KM}$, von Blomberg BME, von dem Borne AEGKR, et al. Neutrophil cytoplasmic antibodies (p-ANCA) in ulcerative colitis. $\mathcal{F}$ Clin Pathol 1994;47:257-62.

16 Billing P, Tahir S, Calfin B, Gagne G, Cobb L, Targan S, et al. Nuclear localization of the antigen detected by ulcerative colitis-associated perinuclear antineutrophil cyulcerative colitis-associated perinuclear antineutrophic

17 Cambridge G, Rampton DS, Stevens TRJ, McCarthy DA, Cambridge G, Rampton DS, Stevens TRJ, McCarthy DA,
Kamm M, Leaker B. Anti-neutrophil antibodies in inflammatory bowel disease: prevalence and diagnostic role. Gut 1992;33:668-74.

18 Tysk C, Järnerot G. Ulcerative proctocolitis in Örebro, Sweden. A retrospective epidemiologic study, 1963-1987. Scand $\mathcal{F}$ Gastroenterol 1992;27:945-50.

19 Bøyum A. Separation of blood leucocytes, granulocytes and lymphocytes. Tissue Antigens 1974;4:269-74.

20 Harlow E, Lane D, eds. Cell staining. In: Antibodies: a laboratory manual. New York: Cold Spring Harbor Laboratory, 1988:359-420.

21 Hopwood D. Fixation and fixatives. In: Bancroft JD, Stevens A, eds. Theory and practice of histological techniques. A, eds. Theory and practice of histological techniques. ingstone, 1990:21-42.

22 Marriot DW, Carlton SJ. Immunofluorescent techniques. In: Bancroft JD, Stevens A, eds. Theory and practice of histological techniques. Edinburgh, London, Melbourne, New York: Churchill Livingstone, 1990:451-63.

23 Spickett GP, Broomhead V. Formalin fixation and patterns of antineutrophil cytoplasmic antibodies. 7 Clin Pathol 1995;48:89-90.

24 Peen E, Almer S, Bodemar G, Rydén B-O, Sjölin C, Tejle K, et al. Anti-lactoferrin antibodies and other types of ANCA in ulcerative colitis, primary sclerosing cholangitis, and Crohn's disease. Gut 1993;34:56-62.

25 Nässberger L, Ljungh A, Schumacher G, Kollberg B. $\beta$-glucuronidase antibodies in ulcerative colitis [letter]. Lancet 1992:340:734-5.

26 Sung JY, Chan KL, Hsu R, Liew CT, Lawton JWM. Ulcerative colitis and antineutrophil cytoplasmic antibodies in Hong Kong Chinese. Am $\mathcal{F}$ Gastroenterol 1993;88:864-9.

27 Deusch K, Oberstadt K, Schaedel W, Weber M, Classen M. P-ANCA as a diagnostic marker in ulcerative colitis. $A d v$ Exp Med Biol 1993;336:527-31.

28 Halbwachs-Mecarelli L, Nusbaum P, Noël LH, Reumaux $D$, Erlinger S, Grünfeld JP, et al. Antineutrophil cytoplasmic antibodies (ANCA) directed against cathepsin G in ulcerative colitis, Crohn's disease and primary sclerosing cholangitis. Clin Exp Immunol 1992;90:79-84.

29 Kaneko K, Suzuki Y, Yamashiro Y, Yabuta K. Is p-ANCA in ulcerative colitis directed against $\beta$-glucuronidase? [letter] Lancet 1993;341:320-3. 